\section{RMD Open}

Rheumatic \&

Musculoskeletal Diseases

\title{
Predictors of response to TNF inhibitors in rheumatoid arthritis: an individual patient data pooled analysis of randomised controlled trials
}

\author{
Johan Law-Wan, ${ }^{1,2}$ Marc-Antoine Sparfel, ${ }^{1,2}$ Sophie Derolez, ${ }^{1,2}$ \\ Nicolas Azzopardi, ${ }^{2,3}$ Philippe Goupille, ${ }^{1,2}$ Jacqueline Detert, ${ }^{4}$ \\ Denis Mulleman (D) , 1,2 Theodora Bejan-Angoulvant (D) 5,6
}

To cite: Law-Wan J, Sparfel M-A, Derolez S, et al. Predictors of response to TNF inhibitors in rheumatoid arthritis: an individual patient data pooled analysis of randomised controlled trials. RMD Open 2021;7:e001882. doi:10.1136/ rmdopen-2021-001882

- Additional supplemental material is published online only. To view, please visit the journal online (http://dx.doi.org/10. 1136/rmdopen-2021-001882).

$\mathrm{JL}-\mathrm{W}$ and M-AS contributed equally.

Received 18 August 2021 Accepted 15 October 2021

\section{Check for updates}

(C) Author(s) (or their employer(s)) 2021. Re-use permitted under CC BY-NC. No commercial re-use. See rights and permissions. Published by BMJ.

For numbered affiliations see end of article.

Correspondence to Prof Denis Mulleman; denis.mulleman@univ-tours.fr

\section{ABSTRACT}

Objective To identify patient characteristics associated with responsiveness to tumour necrosis factor inhibitors (TNFi) in rheumatoid arthritis (RA).

Materials and methods Individual patient data from 29 randomised controlled trials (RCTs) evaluating the efficacy of a TNFi versus placebo or conventional therapy were obtained. Response to treatment was assessed in subgroups according to the following baseline characteristics: smoking status, physical activity, sex, age, body mass index, autoantibody profile, disease duration, high initial disease activity defined by Disease Activity Score on 28 joints (DAS28)(C reactive protein (CRP)) $>5.1$. The primary outcome was the between-treatment group difference in DAS28(CRP) change from baseline to 6 months. The secondary endpoints were the betweentreatment group difference in final DAS28(CRP) measured until 6 months and EULAR response criteria until 6 months. Data from each RCT were then pooled by the MantelHaenszel method using a random effects model. A linear metaregression was also carried out on two data-sharing platforms separately to support the results.

Results Individual data of 11617 patients from 29 RCTs were analysed. Until 6 months, a significantly higher EULAR non-response rate was observed in obese patients (OR 0.52 vs 0.36 for non-obese, $p=0.01$ ). A multivariable regression model performed on 7457 patients indicated that patients treated by TNFi had a final DAS28(CRP) decreased by 0.02 for each year of disease duration $(p<0.001)$, and a 0.21 decreased for patients with a baseline DAS28(CRP) $>5.1(p<0.001)$.

Conclusions In RA, patients who are more responsive to TNFi are those who are non-obese, have a long disease duration and have a high initial disease activity.

\section{INTRODUCTION}

Rheumatoid arthritis (RA) is the most common chronic systemic autoimmune disease with a prevalence of $0.3 \%-1.0 \%{ }^{1}$ Despite considerable progress in the knowledge of its pathogenesis and therapeutic

\section{Key messages}

What is already known about this subject?

- Demographics and disease characteristics may modulate the effect of tumour necrosis factor inhibitors (TNFi).

- Meta analysis based on individual patient data is a reliable and powerful method to analyse factors related to treatment response.

What does this study add?

- High body mass index increased the odds of nonresponse to TNFi in rheumatoid arthritis (RA).

- Patients with RA with high baseline disease activity and those with long disease duration are likely to achieve a better response to TNFi as compared with others.

How might this impact on clinical practice or further developments?

- Bodyweight control of RA is essential when starting a TNFi.

management, ${ }^{2}{ }^{3}$ disease remission or low disease activity is not obtained in all patients. ${ }^{4}$ Tumour necrosis factor inhibitors (TNFi) are the first biological agents available in RA and are still widely used in patients with inadequate response to conventional diseasemodifying antirheumatic drugs (csDMARDs). However, approximately one-third of patients with RA respond insufficiently to TNFi. ${ }^{5-8}$

The reasons behind the heterogeneity in response remain unclear. Demographic, disease-related and environmental factors could contribute to the variability in clinical response to TNFi. Some factors have been associated with a poor response such as smoking, ${ }^{9-12}$ being a woman, ${ }^{10} 1314$ older age, ${ }^{15}{ }^{16}$ obesity, ${ }^{17}$ presence of rheumatoid factor (RF) and anticitrullinated protein 
antibodies (ACPAs),${ }^{18}$ long disease duration ${ }^{13}$ and high disease activity, ${ }^{19-22}$ with conflicting results though. ${ }^{192} 23$ Available data are sparse on the influence of physical activity on the response to TNFi. Physical activity seems to decrease fatigue ${ }^{24}$ and improve quality of life,,${ }^{25}$ but does not seem to decrease inflammation parameters. ${ }^{27}$

We therefore aimed to study the influence of these factors on the effect of TNFi by performing a pooledanalysis of randomised clinical trials that evaluated efficacy of TNFi compared with placebo in subgroups of interest.

\section{METHODS}

\section{Systematic review}

A systematic review of randomised controlled clinical trials was performed according to Preferred Reporting Items for Systematic Reviews and Meta-Analyses guidelines, ${ }^{28}$ with the aim of studying the efficacy of TNFi according to different demographic and disease-related factors. The subgroups of interest were selected according to smoking status, physical activity, sex, age, body mass index (BMI), autoantibody status, disease duration and disease activity score at baseline. The protocol was registered in the PROSPERO database (number CRD42018071079) in January 2018 and was updated in July 2021. We searched for randomised controlled trials (RCTs) and meta-analyses of RCT in Cochrane Central Register of Controlled Trials. This research was performed until January 2017 using the keywords "rheumatoid arthritis", "infliximab", "adalimumab", "etanercept", "golimumab" and "certolizumab". Two authors (TB-A and SD) selected eligible RCT on title and abstract, retrieved full text of eligible articles and decided on the final inclusion. We included RCT comparing TNFi to placebo or csDMARDS in patients with RA, with no restriction on the presence or not of previous TNFi. We excluded non-randomised studies and studies comparing two TNFi without a placebo or csDMARD control groups. If included studies did not report efficacy of TNFi according to subgroups of interest, we sought to obtain individual patient data (IPD).

\section{Data collection}

Given the unavailability of published subgroup of interest analyses, we contacted the corresponding authors and/ or sponsors of these trials in order to obtain aggregated data and/or IPD and to perform a pooled analysis. Since most of the data were stored securely on data-sharing platforms, we requested each of these platforms an access to the raw dataset. We signed data use agreements for two platforms, Yale Open Data Access (YODA) and Vivli Centre for Global Clinical Research, that allowed us to access IPD for 26 trials. We further obtained IPD for two AMGEN trials and for one academic trial (online supplemental table 1).

\section{Subgroup of interest}

After obtaining access to data, we analysed the efficacy of TNFi, regardless of the dose used, compared with control in subgroups of interest. These subgroups were smoking status (never/ever smokers), current physical activity (yes/no), sex (men/women), age ( $\leq 50 />50$ years), BMI $\left(<30 / \geq 30 \mathrm{~kg} / \mathrm{m}^{2}\right)$, RF status (positive/negative), ACPA status (positive/negative), RA disease duration $(<2 / 2$ to $10 / \geq 10$ years) and baseline DAS28(CRP) $(\leq 5.1 />5.1)$.

\section{Outcomes}

The predefined primary endpoint was ACR20 score after 6 months of follow-up, and secondary endpoints were ACR50, ACR70, DAS28(CRP) and DAS28(ESR). Due to the impossibility to obtain ACR response from the raw dataset for eight trials, we decided in November 2019 to modify the primary endpoint to between-treatment group differences in DAS28(CRP) change ( $\triangle \mathrm{DAS} 28(\mathrm{CRP})$ ) from baseline to 6 months (or as close as 6 months, depending on each trial available data). Secondary endpoints were between-treatment group differences in final DAS28(CRP) measured at 6 months (or as close as 6 months) and EULAR response criteria at 6 months (or as close as 6 months) ${ }^{29}$ EULAR response criteria were used to stratify the groups of response, that is, good response if final DAS28 was $\leq 3.2$ with a DAS28 improvement from baseline of at least 1.2 points; non-response if final DAS28 was $>5.1$ with a DAS28 improvement from baseline $\leq 1.2$ points or DAS28 improvement from baseline $\leq 0.6$ points, and moderate response if DAS28 did not meet these criteria. For trials with missing erythrocyte sedimentation rate (ESR), DAS28(CRP) was used to categorise EULAR response using the same cut-offs as for DAS28(ESR).

\section{Statistical analyses}

Statistical analyses were performed using R Studio software. Descriptive results are presented as median (minmax) or mean (IQR) unless stated otherwise. Pooled ORs or mean difference with $95 \%$ CIs for EULAR response and for DAS28(CRP) differences, respectively, between TNFi and placebo were calculated using two-step metaanalyses. First, aggregate data regarding treatment response in each subgroup of interest were estimated from IPD. Second, a random-effect Mantel-Haenszel model was applied to calculate pooled effect. We considered a significant difference between subgroups if the $p$ value is $<0.05$. Between-study heterogeneity was quantified using Cochrane $Q$ and $I^{2}$ statistics. Heterogeneity was considered low if $\mathrm{I}^{2}$ was $<30 \%$, moderate if $\mathrm{I}^{2}$ was $30 \%-50 \%$, substantial if $\mathrm{I}^{2}$ was $50 \%-70 \%$ and considerable if $\mathrm{I}^{2}$ was $>70 \%$. IPD from YODA and Vivli platforms were used to perform two separate linear metaregressions with final DAS28(CRP) as independent variable adjusted on baseline DAS28(CRP), trial and treatment by subgroup variable interaction (bivariate analyses). Multivariable metaregressions of final DAS28(CRP) adjusted on baseline DAS28(CRP), trial, age and subgroup variables with a $p$ value for interaction of $<0.20$ in bivariate analyses were also performed.

The analysis was performed following the intentionto-treat principle, with no replacement of missing data. 


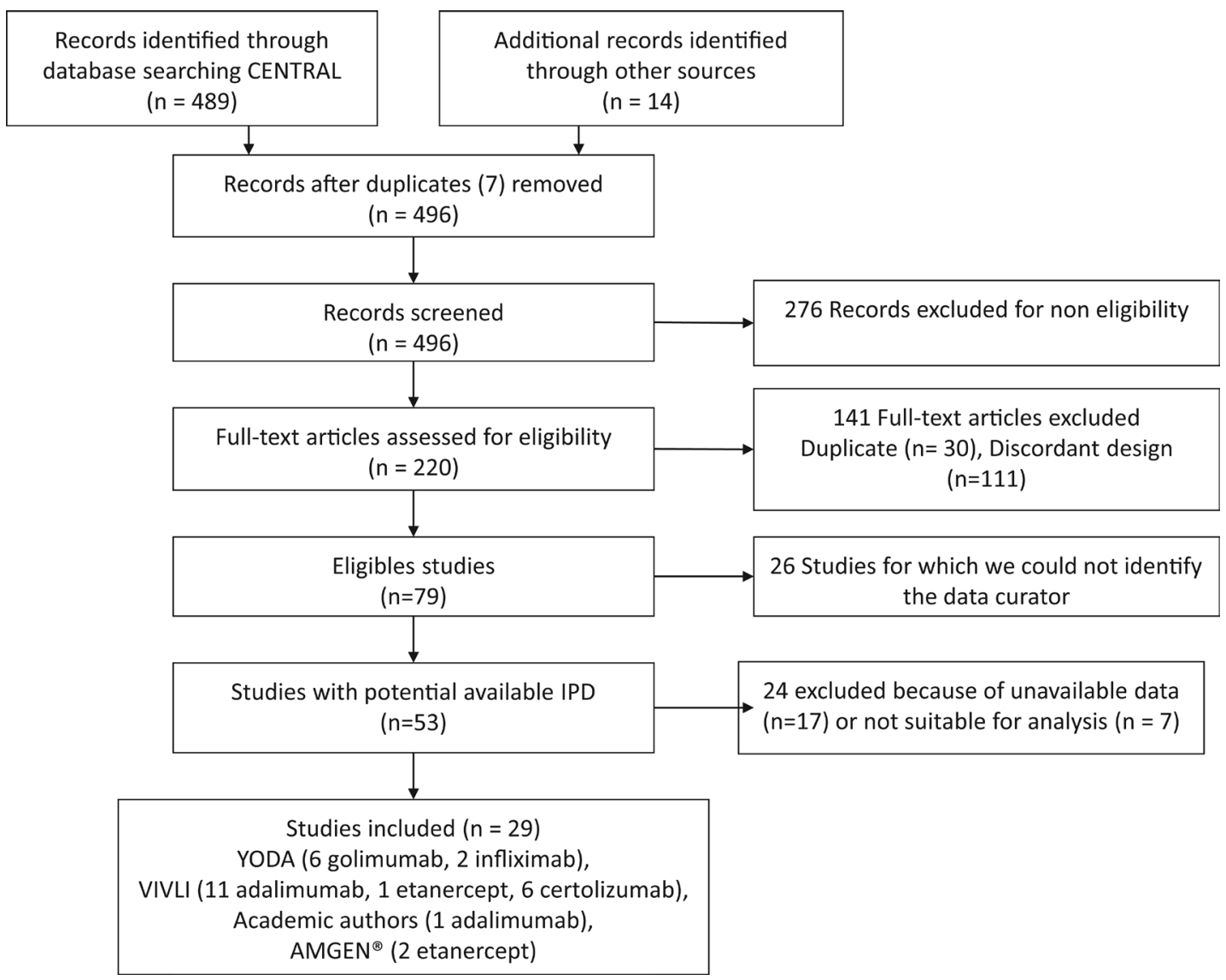

Figure 1 Flow diagram of studies selection process. YODA, Yale Open Data Access.

Since this was an exploratory post hoc analysis, no $\mathrm{p}$ value adjustment for multiplicity was performed. Therefore, results should be considered with caution.

\section{RESULTS}

\section{Search process}

We found 496 articles published between 1994 and 2017, and 220 of them were eligible after selection on title and abstract, and 79 fulfilled all inclusion and exclusion criteria (figure 1). At the end of our search, we did not retrieve any RCT that reported aggregated results regarding the efficacy of TNFi in the subgroups of interest. We therefore retrieved IPD for 29 trials. As to June 2021, we obtained access data for 8 trials from the YODA platform, 18 trials from the Vivli platform, 2 trials from AMGEN and 1 trial from an academic author (figure 1).

\section{Characteristics of included clinical trials}

This pooled analysis included 29 RCTs evaluating five TNFi: 12 evaluated adalimumab; 3 evaluated etanercept; 6 evaluated certolizumab; 6 evaluated golimumab; and 2 evaluated infliximab (table 1 and online supplemental table 1). All RCTs were double-blinded, placebocontrolled, parallel group trials conducted between 1997 and 2015. The median number of patients randomised was 444 (range: 47-1648). Individual data of 14838 randomised patients were available. Clinical and biological data were missing in some studies, leading to 11617 (78\%) IPDs available for analyses. Physical activity data were missing for 25 studies. Some data could not be retrieved for confidentiality and anonymisation reasons, such as patients' age in six certolizumab studies and disease duration in four certolizumab studies that reported these data as intervals. Treatment response could be evaluated at week 24 for 21 trials (72\%), at week 26 for 2 trials (7\%), at week 30 for 2 trials $(7 \%)$, and at week 12 for 4 trials $(14 \%)$.

\section{Difference in $\triangle \mathrm{DAS28}$ (CRP) between baseline and until 6 months}

Smoking status, physical activity, sex, age, BMI, ACPA, RF status, disease duration and baseline DAS28(CRP) did not significantly influence the difference in $\triangle \mathrm{DAS} 28$ (CRP) between TNFi and placebo in subgroup analyses ( $p>0.05$ for subgroup differences, tables 2 and 3). Heterogeneity was considerable $\left(\mathrm{I}^{2}>70 \%\right)$ in some subgroups: nonsmoking patients, women, non-obese patients, patients with RF and ACPA positive status and patients with high baseline DAS28(CRP).

\section{Difference in final DAS28(CRP)}

Smoking status, physical activity, sex, age, BMI, ACPA and RF status, disease duration and baseline DAS28(CRP) did not significantly influence the final DAS28(CRP) between 


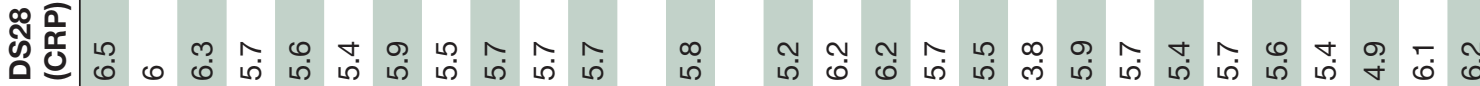

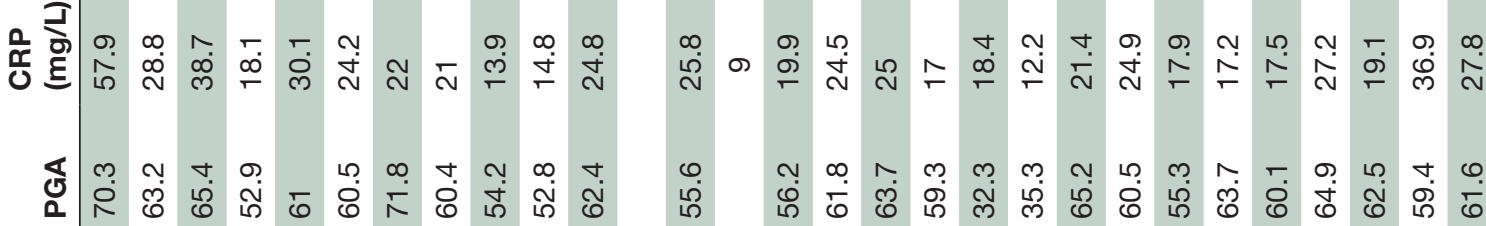
등 픈

产

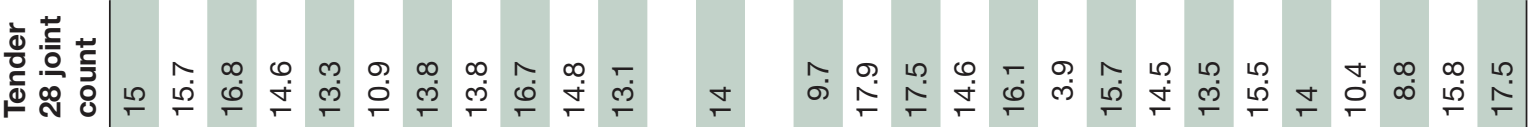

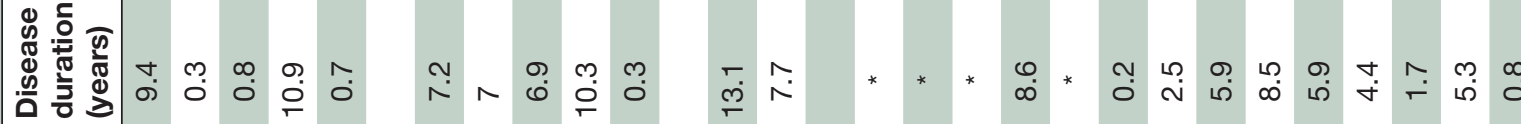

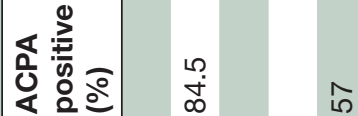

$\infty \stackrel{\sim}{\infty}$

$\stackrel{\Upsilon}{\text { ஸิ }}$

œ

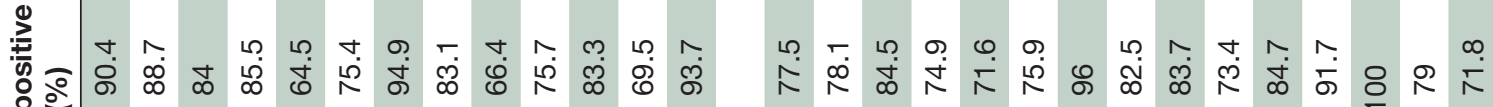

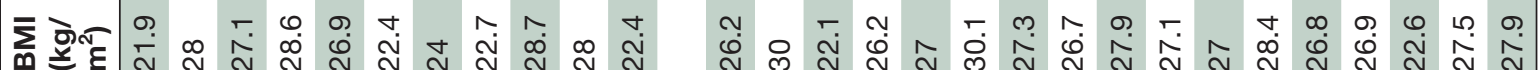

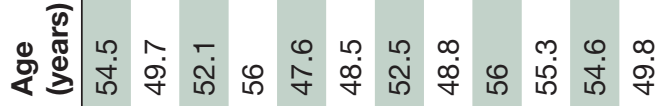

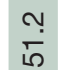

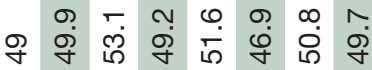

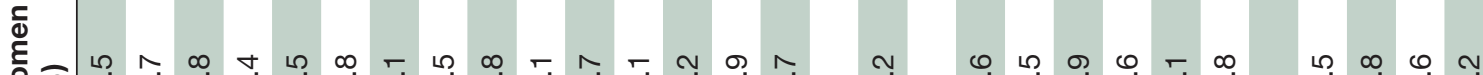

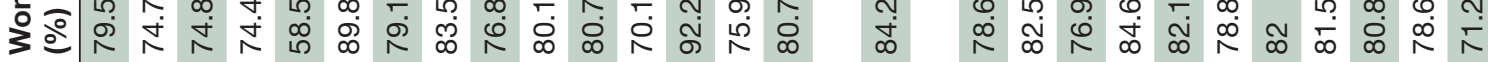

$\begin{array}{lll}2 & 0 & 0\end{array}$ $\stackrel{\infty}{\infty} \stackrel{\infty}{\sim} \stackrel{\infty}{\sim}$

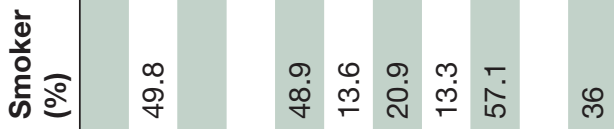

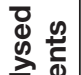

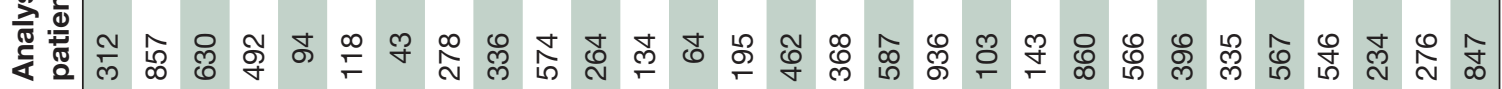

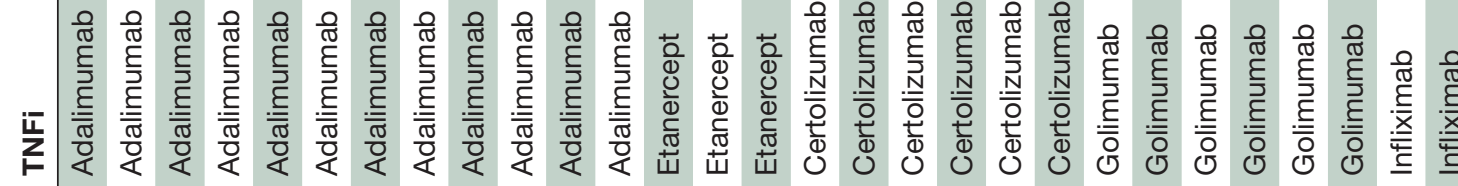

万ิ సิ

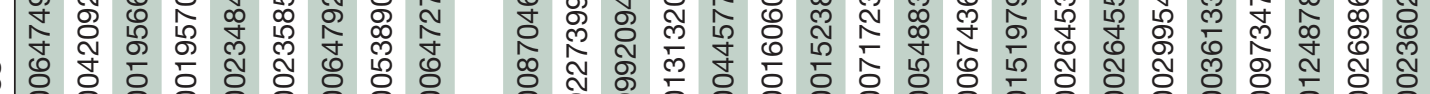
ঋ

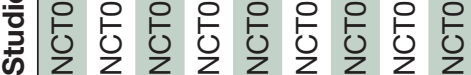

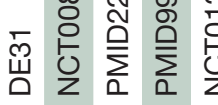

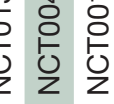

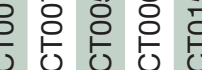

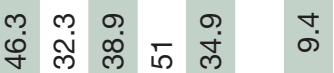

ฮั

중.으

응

之ं Ф

m

ㅊํำ

的并芒

का $\frac{1}{\Phi} . \frac{5}{0}$

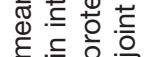

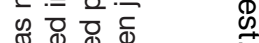

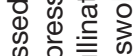

के $\frac{2}{x} \overline{5}$

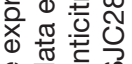

สั

ฮั 政 
Table 2 MD in $\triangle \mathrm{DAS} 28(\mathrm{CRP})$ between baseline and until 6 months by clinical and biological baseline characteristics

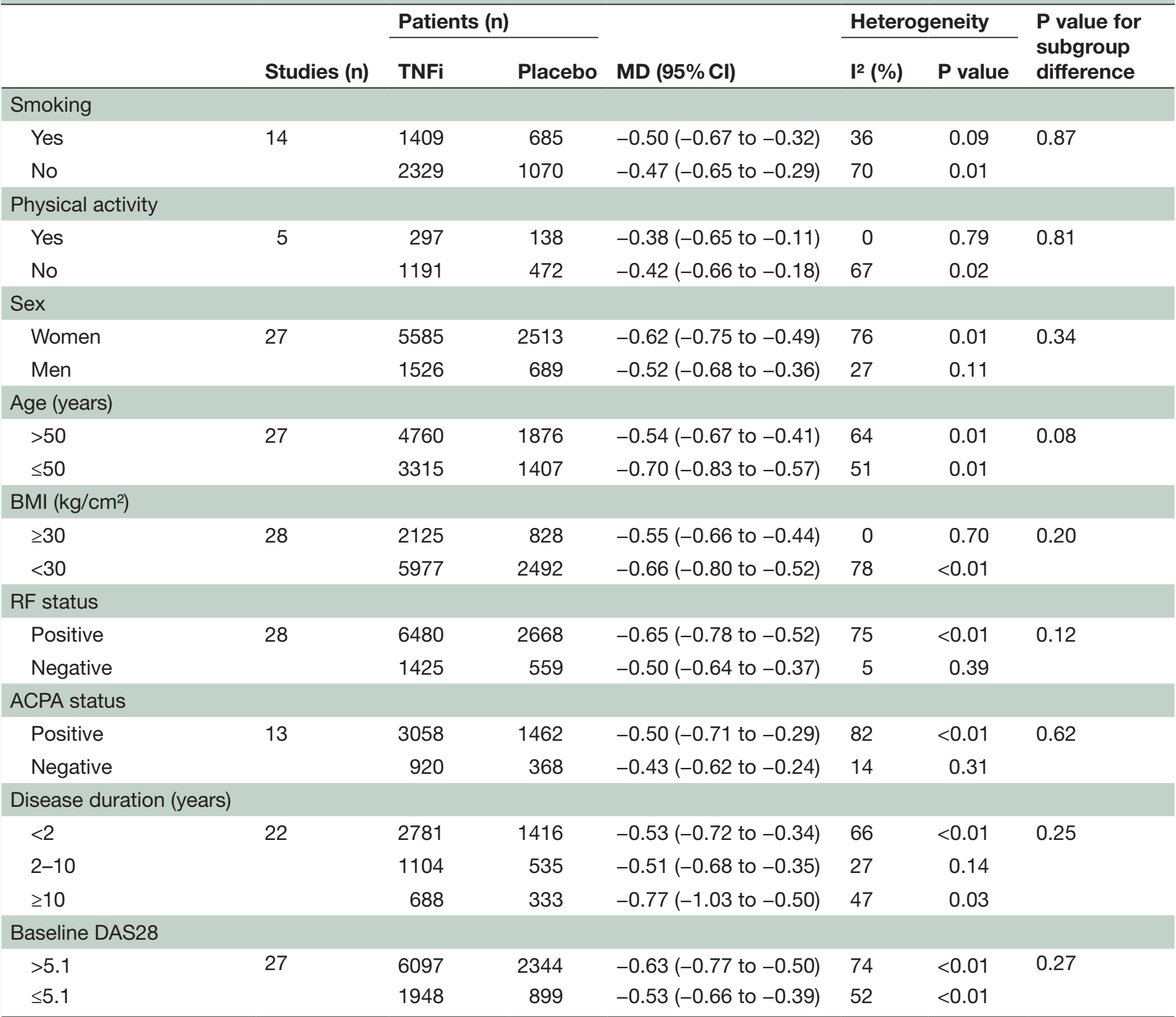

ACPA, anticitrullinated protein antibody; BMI, bodymass index; CRP, C reactive protein; Baseline DAS28, DAS28(CRP) score at baseline; DAS28, Disease Activity Score on 28 joints; $\triangle \mathrm{DAS28(CRP),} \mathrm{DAS28(CRP)} \mathrm{change;} \mathrm{MD,} \mathrm{mean} \mathrm{difference;} \mathrm{RF,} \mathrm{rheumatoid} \mathrm{factor;} \mathrm{TNFi,} \mathrm{tumour}$ necrosis factor inhibitors.

TNFi and placebo in subgroup meta-analyses ( $\mathrm{p}>0.05$ for subgroup differences, online supplemental table 2).

\section{Good EULAR response}

Smoking status, physical activity, sex, age, BMI, ACPA or RF status, disease duration and baseline DAS28 did not significantly influence the good EULAR response between TNFi and placebo in subgroup meta-analyses ( $>0.05$, online supplemental table 3$)$.

\section{EULAR non-response}

We observed a qualitative and significant influence of obesity on the odd of being EULAR non-responder between TNFi and placebo (online supplemental table 4 ). Obese patients had a higher risk of non-response (OR $0.52,95 \%$ CI 0.43 to 0.63 ) compared with patients with a
BMI of $<30$ (OR $0.36,95 \%$ CI 0.30 to 0.45 ), with $\mathrm{p}=0.01$ for subgroup difference (table 3 and online supplemental figure 1). There was no influence of other covariates (smoking status, physical activity, sex, age, baseline DAS28(CRP), disease duration, ACPA and RF status) on the odds of non-response between TNFi and placebo ( $p>0.05$ for subgroup differences).

\section{Metaregression analyses}

Bivariable and multivariable metaregression results are shown in table 4. Metaregressions were performed on 7457 patients (18 RCTs) from Vivli and on 3767 (8 RCTs) patients from YODA. Bivariate analyses indicated that being treated by TNFi was associated with a significantly lower final DAS28(CRP), while a higher baseline 
Table $3 \quad P$ values for subgroup difference for $\triangle \mathrm{DAS28}(\mathrm{CRP})$, final DAS28(CRP), good EULAR response and non-EULAR response between TNFi and placebo

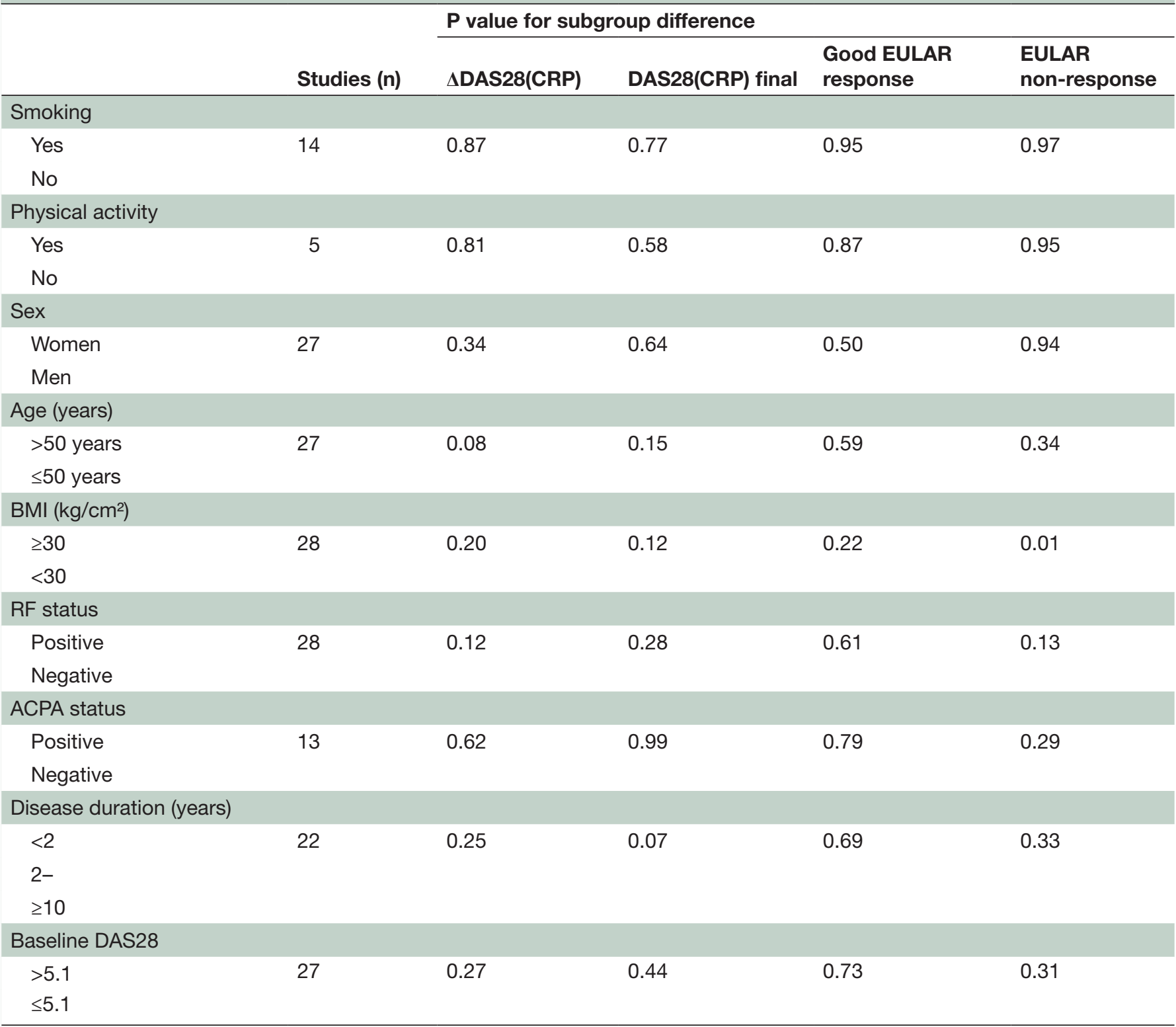

ACPA, anticitrullinated protein antibody; BMI, body mass index; CRP, C reactive protein; Baseline DAS28, DAS28(CRP) score at baseline; DAS28, Disease Activity Score on 28 joints; $\triangle \mathrm{DAS28(CRP),} \mathrm{DAS28(CRP)} \mathrm{change;} \mathrm{RF,} \mathrm{rheumatoid} \mathrm{factor;} \mathrm{TNFi,} \mathrm{tumour} \mathrm{necrosis} \mathrm{factor}$ inhibitors.

DAS28(CRP) was associated with a significantly higher final DAS28(CRP) in trials from both databases. Men presented a lower final DAS28(CRP) than women, and disease duration was associated with an increased final DAS28(CRP) in trials from the Vivli database. Significant treatment effect modifiers in bivariate analyses were disease duration with a lower $0.02 /$ year (in trials from the Vivli database) and baseline DAS28(CRP) with a higher $0.1 /$ baseline unit (in trials from YODA database) final DAS28(CRP) in treated patients.

Four variables, that is, age, sex, BMI and disease duration, were included in the multivariable models. Using the individual data from Vivli $(\mathrm{n}=7457)$, the model indicated that disease duration as a continuous variable and baseline DAS28(CRP) as a categorial variable significantly modified treatment effect on final DAS28(CRP). Patients treated by TNFi had a final DAS28(CRP) decreased by 0.02 for each year of disease duration $(p<0.001)$, and a 0.21 decreased for patients with a baseline DAS28 $($ CRP $)>5.1 \quad(p=0.05)$. These results were not observed in the metagression performed on the 3767 patients from the YODA platform.

\section{DISCUSSION}

From the meta-analysis on pooled data, the sole characteristic associated with a clinical outcome was BMI, which increased the odds of being non-responders. According 
Table 4 Bivariate and multivariate metaregression analyses for until 6 months DAS28(CRP)

\begin{tabular}{|c|c|c|c|c|c|c|}
\hline & \multicolumn{3}{|c|}{ YODA platform (3767 patients) } & \multicolumn{3}{|c|}{ Vivli platform (7457 patients) } \\
\hline & $\begin{array}{l}\text { Standardised } \\
\text { coefficient }\end{array}$ & SD & $P$ value & $\begin{array}{l}\text { Standardised } \\
\text { coefficient }\end{array}$ & SD & $P$ value \\
\hline \multicolumn{7}{|l|}{ Bivariate } \\
\hline Smoking & 0.051 & 0.09 & 0.58 & 0.09 & 0.08 & 0.27 \\
\hline Exercise & -0.23 & 0.12 & 0.06 & & & \\
\hline Men & -0.15 & 0.09 & 0.12 & -0.26 & 0.07 & $<0.001$ \\
\hline $\mathrm{Age}^{*}$ & 0.003 & 0.003 & 0.28 & -0.003 & 0.002 & 0.17 \\
\hline $\mathrm{BMI}^{*}$ & 0.002 & 0.006 & 0.75 & 0.015 & 0.004 & $<0.001$ \\
\hline $\mathrm{RF}+$ & -0.15 & 0.10 & 0.15 & 0.40 & 1.22 & 0.74 \\
\hline $\mathrm{ACPA}+$ & -0.18 & 0.11 & 0.11 & 0.27 & 0.29 & 0.36 \\
\hline Disease duration* $^{*}$ & 0.005 & 0.01 & 0.70 & 0.013 & 0.005 & $<0.001$ \\
\hline Baseline DAS28(CRP) & 0.54 & 0.04 & $<0.001$ & 0.56 & 0.03 & $<0.001$ \\
\hline Treatment & -0.47 & 0.04 & $<0.001$ & -0.63 & 0.03 & $<0.001$ \\
\hline \multicolumn{7}{|l|}{ Treatment by covariate interaction } \\
\hline Treatment: smoking & -0.003 & 0.11 & 0.97 & 0.02 & 0.10 & 0.81 \\
\hline Treatment: exercise & 0.054 & 0.14 & 0.71 & & & \\
\hline Treatment: men & -0.11 & 0.11 & 0.32 & 0.14 & 0.08 & 0.08 \\
\hline Treatment: age & -0.0005 & 0.004 & 0.89 & 0.006 & 0.003 & 0.06 \\
\hline Treatment: BMI & 0.006 & 0.0077 & 0.43 & 0.002 & 0.005 & 0.63 \\
\hline Treatment: RF+ & 0.02 & 0.12 & 0.86 & 0.85 & 1.34 & 0.53 \\
\hline Treatment: ACPA+ & 0.12 & 0.13 & 0.33 & 0.04 & 0.32 & 0.89 \\
\hline Treatment: duration & 0.01 & 0.015 & 0.41 & -0.02 & 0.005 & $<0.001$ \\
\hline Treatment: baseline DAS28(CRP) & 0.1 & 0.04 & 0.03 & -0.04 & 0.03 & 0.22 \\
\hline \multicolumn{7}{|l|}{ Multivariate $†$} \\
\hline Treatment: duration & 0.02 & 0.02 & 0.32 & -0.02 & 0.006 & $<0.001$ \\
\hline Treatment: baseline DAS28(CRP) & 0.08 & 0.01 & 0.44 & -0.02 & 0.04 & 0.7 \\
\hline $\begin{array}{l}\text { Treatment: baseline } \\
\text { DAS28(CRP) }>5.1\end{array}$ & 0.33 & 0.23 & 0.15 & -0.21 & 0.11 & 0.049 \\
\hline
\end{tabular}

${ }^{*}$ For one unit (year, $\mathrm{kg} / \mathrm{m}^{2}$ ).

†Sex, age, BMI and disease duration.

ACPA, anticitrullinated protein antibody; BMI, body mass index; CRP, C reactive protein; Baseline DAS28, DAS28(CRP) score at baseline;

DAS28, Disease Activity Score on 28 joints; RF, rheumatoid factor; YODA, Yale Open Data Access.

to the metaregression analysis based on individual data, the multivariable model found that disease duration and baseline DAS28(CRP) categories interacted with the final DAS28(CRP). In the present work, we did not find any influence of smoking status, physical activity, sex, age, RF or ACPA status on response to TNFi, which was in accordance to previous meta-analyses ${ }^{23}$ and registries ${ }^{21}$ but not with other retrospective cohorts. ${ }^{1923}$

A recent meta-analysis showed a lower minimal disease activity achievement in obese patients with psoriatic arthritis or RA compare with those normal BMI. ${ }^{30}$ One hypothesis would be a decrease of TNFi concentration in obese patients due to a larger volume of distribution which is increased with body size. ${ }^{31} 32$ Therefore, an increase in the dosage of TNFi in obese patients could be considered. However, it has also been observed in the literature that obese patients treated with infliximab, whose dosage is based on body weight, have a lesser response ${ }^{17}$ suggesting the role of adipose tissue. It has indeed been demonstrated that adipose tissue can produce adipokines, capable of inducing the production of proinflammatory cytokines such as tumour necrosis factor or interleukin- $6,{ }^{33}$ which may explain the lesser response to TNFi in obese patients. Furthermore, interaction between obesity and other factors such as physical activity may exist in the clinical trials included. ${ }^{34}$ However, because of lack of information on physical activity in most of trials, such correlations could not be studied.

We observed that patients with long disease duration, that is, $\geq 10$ years, seemed to have a better response than others, which was not reported in the literature so far. In clinical practice, patients with long disease duration who require TNFi are usually those who have already failed to various treatments, which is known as a poor 
prognostic factor. ${ }^{13}$ This surprising result was seen in one of the data-sharing platforms and was not attributed to age nor disease activity. Thus, patients with long-standing disease with insufficient control of disease activity are as eligible as those with shorter disease duration, with even an additional effect of TNFi.

High baseline disease activity, that is, DAS28(CRP) of $>5.1$, was predictive of a favourable, although small, additional reduction in DAS28(CRP) at the time of 3 and 6 months, as compared with patients with moderate disease activity. It seems coherent that patients with the highest inflammatory burden obtain a tangible effect as compared with those with less inflammation, in which the disease improvement is less pronounced. This finding was only observed by the metaregression analysis issued from one datasharing platform. The meta-analysis of pooled data yielded no statistically significant difference but pointed to the same direction.

Our study has some strengths that deserve to be mentioned. This is the first analysis based on a large amount of IPD, studying the effect of demographic and disease factors on response to TNFi in RA, which allowed us to increase the power to show a very small difference between some subgroups of patients. The current knowledge are predominantly based on national registries, retrospective cohorts or aggregate data meta-analyses. The use of IPD from data-sharing platforms enables reusing raw data from a substantial number of studies. Furthermore, in comparison with meta-analyses based on aggregated data published by the investigators, obtaining raw data allowed us to study in a standardised manner various parameters and to pool them together, creating a large database. The results obtained here have important clinical impacts in the context of personalised treatment strategy, for example, to increase awareness on negative predictive factors such as obesity when initiating a TNFi.

The main challenge in our work was the data selection and availibility. In most cases, we chose to exclude from the subgroup analysis patients who could not be categorised, which may have caused bias in the analysis. ${ }^{35}$ Some adjustments were nevertheless made to limit this loss of data. For instance, we adapted the age intervals provided by the sponsor to our population subgroups, seeking for the best compromise (online supplemental table 5). Similarly, the absence of ESR in some studies led us to use DAS28(CRP) and $\triangle$ DAS28(CRP) in the categorisation of responder and non-responder patients instead of DAS28(ESR) and $\triangle$ DAS28(ESR), which may have somehow overestimated the EULAR response rate. ${ }^{36}$

Another limitation of our study is the different time points of response assessment, that is, at week 30 for 2 studies, at week 24 for 21 studies, at week 26 for 2 studies and at week 12 for 4 of them (online supplemental tables 6 and 7 ). At the time of the protocol writing, the 6-month time point was selected to capture all patients with sustained clinical response, to discriminate from those with early transient response, sometimes related to placebo effect. For studies where evaluation at 6 months was not available, we chose the 3 -month time point. We made a compromise in order to include as many patients as possible in the analysis and to stick to the clinical relevance. In the treat-totarget strategy, ${ }^{4}$ European recommendations allow and require the clinician to evaluate the response treatment as early as 3 months.

For all the studies, we categorised treatment arms into two groups, either placebo or TNFi, whereas there were often several different TNFi groups with different dosages. In case of different treatment groups, all dosing regimens were gathered and compared with placebo. This did not hamper our conclusions, which was to compare the magnitude of response according to demographics and diseases characteristics, not according to the dosing regimen.

We did not plan to study the influence of concomitant treatments such as methotrexate, because the effect of such treatments is already known. Our objective was to study other treatment effect modifiers. Since such concomitant treatments were equally distributed between treatment arms, we considered that their influence on the effect of other covariate was unlikely. We did not analyse the dose of TNFi. The dose-response relationship would deserve a specific attention and further studies but was not the objective of this study. Because of the randomisation, we considered that this would not hamper our conclusions.

Finally, we should acknowledge the limitation of clinical trials and the extrapolation of our conclusions to patients seen in clinical practice. Differences in patients included in clinical trials, duration of treatment and follow-up, and the fact that we did not study the effect of TNFi monotherapy compared with their use in association with methotrexate could lead to differences in extrapolation to clinical practice. However, clinical trials remain the gold standard to assess treatment efficacy and the influence of treatment effect modifiers.

In conclusion, this meta-analysis on individual patients with RA data confirmed that obese patients are less responsive to TNFi as compared with non-obese patients. Those with long disease duration and those with a high baseline disease activity achieve a better response than others.

\section{Author affiliations}

${ }^{1}$ Department of Rheumatology, CHRU de Tours, Tours, France

${ }^{2}$ EA 7501 GICC, Université de Tours, Tours, France

${ }^{3}$ ERL 7001, CNRS, Tours, France

${ }^{4}$ Medical Practice of Rheumatology and Clinical Immunology, Rheumatologischimmunologische Praxis, Templin, Germany

${ }^{5}$ Department of Clinical Pharmacology, CHRU de Tours, Tours, France

${ }^{6}$ EA 4245 T2I, Université de Tours, Tours, France

Acknowledgements We thank the University of Tours for assistance with data use agreement. We are indebted to AMGEN and the Yale Open Data Access team for providing access to data. This publication is based on research using data from 
data contributors AbbVie, Pfizer and UCB that have been made available through Vivli. Vivli has not contributed to or approved, and is not in any way responsible for the contents of this publication. We thank Professor Laure Gossec and Professor Cécile Gaujoux-Viala for offering their advice.

Contributors DM coordinated the work, participated in the interpretation of the analyses and helped draft the manuscript. TB-A initiated the project, performed the statistical analyses and helped draft the manuscript. SD wrote the protocol of the meta-analysis, selected the studies and asked for data. M-AS and JL-W ensured obtainment of data, analysed the results and drafted the manuscript. NA handled the database and helped in improving the manuscript. PG contributed to the interpretation of the results and manuscript improvement. JD provided individual patient data from the PMID 22739990 study evaluating adalimumab and helped in improving the manuscript. All authors read and approved the final version of the manuscript. DM is the guarantor for this work.

Funding This work was partly supported by the French Higher Education and Research Ministry under the program Investissements d'avenir (grant agreement: LabEx MAblmprove ANR-10-LABX-53-01).

Competing interests $P G$ participated on behalf of his institution in clinical trials sponsored by Abbvie, Roche, BMS, Boehringer, Lilly, Novartis, Pfizer, UCB, Janssen and MSD; acted as a consultant and given lectures for Abbvie, Biogaran, BMS Hospira, Janssen, MSD, Pfizer, Sanofi-Genzyme and UCB; had been invited to attend international congresses by MSD, Roche, BMS and Abbvie. DM acted as a consultant and gave lectures on behalf of his institution for Pfizer, Novartis and Grifols; had been invited to attend an international congress by Janssen-Cilag, GSK and Chugai. His institution received grants for research from the non-governmental organisation Lions Club Tours Val de France. M-AS, JL-W, SD, JD and TB-A declared no conflict of interest in relation with the present work.

Patient consent for publication Not applicable.

Provenance and peer review Not commissioned; externally peer reviewed.

Data availability statement Data may be obtained from a third party and are not publicly available. The raw data were not extracted but analysed remotely from the Yale Open Data Access and Vivli platforms.

Open access This is an open access article distributed in accordance with the Creative Commons Attribution Non Commercial (CC BY-NC 4.0) license, which permits others to distribute, remix, adapt, build upon this work non-commercially, and license their derivative works on different terms, provided the original work is properly cited, appropriate credit is given, any changes made indicated, and the use is non-commercial. See: http://creativecommons.org/licenses/by-nc/4.0/.

\section{ORCID iDs}

Denis Mulleman http://orcid.org/0000-0003-4089-7513

Theodora Bejan-Angoulvant http://orcid.org/0000-0002-0018-9996

\section{REFERENCES}

1 Silman AJ, Pearson JE. Epidemiology and genetics of rheumatoid arthritis. Arthritis Res 2002;4:S265-72.

2 McInnes IB, Schett G. The pathogenesis of rheumatoid arthritis. N Engl J Med 2011;365:2205-19.

3 Smolen JS, Aletaha D, Mclnnes IB. Rheumatoid arthritis. Lancet 2016;388:2023-38.

4 Smolen JS, Landewé RBM, Bijlsma JWJ, et al. EULAR recommendations for the management of rheumatoid arthritis with synthetic and biological disease-modifying antirheumatic drugs: 2019 update. Ann Rheum Dis 2020;79:685-99.

5 Gibbons LJ, Hyrich KL. Biologic therapy for rheumatoid arthritis: clinical efficacy and predictors of response. BioDrugs 2009;23:111-24.

6 Hetland ML, Christensen IJ, Tarp U, et al. Direct comparison of treatment responses, remission rates, and drug adherence in patients with rheumatoid arthritis treated with adalimumab, etanercept, or infliximab: results from eight years of surveillance of clinical practice in the nationwide Danish DANBIO registry. Arthritis Rheum 2010;62:22-32.

7 Keystone EC, Kavanaugh AF, Sharp JT, et al. Radiographic, clinical, and functional outcomes of treatment with adalimumab (a human anti-tumor necrosis factor monoclonal antibody) in patients with active rheumatoid arthritis receiving concomitant methotrexate therapy: a randomized, placebo-controlled, 52-week trial. Arthritis Rheum 2004;50:1400-11.

8 Weinblatt ME, Kremer JM, Bankhurst AD, et al. A trial of etanercept, a recombinant tumor necrosis factor receptor:Fc fusion protein, in patients with rheumatoid arthritis receiving methotrexate. $N$ Engl $J$ Med 1999;340:253-9.
9 Canhão H, Rodrigues AM, Dinis de Sousa R, et al. EpiDoC Unit - a clinical research unit open to the rheumatology community. Acta Reumatol Port 2021;46:5-6.

10 Hyrich KL, Watson KD, Silman AJ, et al. Predictors of response to anti-TNF-alpha therapy among patients with rheumatoid arthritis: results from the British Society for rheumatology biologics register. Rheumatology 2006;45:1558-65.

11 Saevarsdottir S, Wedrén S, Seddighzadeh M, et al. Patients with early rheumatoid arthritis who smoke are less likely to respond to treatment with methotrexate and tumor necrosis factor inhibitors: observations from the epidemiological investigation of rheumatoid arthritis and the Swedish rheumatology register cohorts. Arthritis Rheum 2011;63:26-36.

12 Söderlin MK, Petersson IF, Geborek P. The effect of smoking on response and drug survival in rheumatoid arthritis patients treated with their first anti-TNF drug. Scand J Rheumatol 2012;41:1-9.

13 Anderson JJ, Wells G, Verhoeven AC, et al. Factors predicting response to treatment in rheumatoid arthritis: the importance of disease duration. Arthritis Rheum 2000;43:22-9.

14 Jawaheer D, Olsen J, Hetland ML. Sex differences in response to anti-tumor necrosis factor therapy in early and established rheumatoid arthritis - results from the DANBIO registry. $J$ Rheumatol 2012;39:46-53.

15 Mancarella L, Bobbio-Pallavicini F, Ceccarelli F, et al. Good clinical response, remission, and predictors of remission in rheumatoid arthritis patients treated with tumor necrosis factor-alpha blockers: the GISEA study. J Rheumatol 2007;34:1670-3.

16 Radovits BJ, Kievit W, Fransen J, et al. Influence of age on the outcome of antitumour necrosis factor alpha therapy in rheumatoid arthritis. Ann Rheum Dis 2009;68:1470-3.

17 Klaasen R, Wijbrandts CA, Gerlag DM, et al. Body mass index and clinical response to infliximab in rheumatoid arthritis. Arthritis Rheum 2011:63:359-64.

18 Potter C, Hyrich KL, Tracey A, et al. Association of rheumatoid factor and anti-cyclic citrullinated peptide positivity, but not carriage of shared epitope or PTPN22 susceptibility variants, with anti-tumour necrosis factor response in rheumatoid arthritis. Ann Rheum Dis 2009;68:69-74.

19 Atzeni F, Antivalle M, Pallavicini FB, et al. Predicting response to anti-TNF treatment in rheumatoid arthritis patients. Autoimmun Rev 2009;8:431-7.

20 lannone F, Gremese E, Gallo G, et al. High rate of disease remission in moderate rheumatoid arthritis on etanercept therapy: data from GISEA, the Italian biologics register. Clin Rheumatol 2014;33:31-7.

21 Kristensen LE, Kapetanovic MC, Gülfe A, et al. Predictors of response to anti-TNF therapy according to ACR and EULAR criteria in patients with established RA: results from the South Swedish arthritis treatment group register. Rheumatology 2008;47:495-9.

22 Listing J, Strangfeld A, Rau R, et al. Clinical and functional remission: even though biologics are superior to conventional DMARDs overall success rates remain low-results from RABBIT, the German biologics register. Arthritis Res Ther 2006;8:R66.

23 Lv Q, Yin Y, Li X, et al. The status of rheumatoid factor and anticyclic citrullinated peptide antibody are not associated with the effect of anti-TNF $\alpha$ agent treatment in patients with rheumatoid arthritis: a meta-analysis. PLoS One 2014;9:e89442.

24 Cramp F, Hewlett S, Almeida C, et al. Non-pharmacological interventions for fatigue in rheumatoid arthritis. Cochrane Database Syst Rev 2013:CD008322.

25 Prioreschi A, Hodkinson B, Avidon I, et al. The clinical utility of accelerometry in patients with rheumatoid arthritis. Rheumatology 2013;52:1721-7

26 Stenström $\mathrm{CH}$, Minor MA. Evidence for the benefit of aerobic and strengthening exercise in rheumatoid arthritis. Arthritis Rheum 2003;49:428-34.

27 Burghardt R-D, Kazim MA, Rüther W, et al. The impact of physical activity on serum levels of inflammatory markers in rheumatoid arthritis: a systematic literature review. Rheumatol Int 2019;39:793-804.

28 Stewart LA, Clarke M, Rovers M, et al. Preferred reporting items for systematic review and meta-analyses of individual participant data: the PRISMA-IPD statement. JAMA 2015;313:1657-65.

29 van Gestel AM, Prevoo ML, van 't Hof MA, et al. Development and validation of the European League against rheumatism response criteria for rheumatoid arthritis. Comparison with the preliminary American College of rheumatology and the world health Organization/International League against rheumatism criteria. Arthritis Rheum 1996;39:34-40.

30 Lupoli R, Pizzicato P, Scalera A, et al. Impact of body weight on the achievement of minimal disease activity in patients with rheumatic diseases: a systematic review and meta-analysis. Arthritis Res Ther 2016;18:297. 
31 Ternant D, Bejan-Angoulvant T, Passot C, et al. Clinical pharmacokinetics and pharmacodynamics of monoclonal antibodies Approved to treat rheumatoid arthritis. Clin Pharmacokinet 2015;54:1107-23.

32 Ternant D, Ducourau E, Perdriger A, et al. Relationship between inflammation and infliximab pharmacokinetics in rheumatoid arthritis. Br J Clin Pharmacol 2014:78:118-28.

33 Ellulu MS, Patimah I, Khaza'ai $\mathrm{H}$, et al. Obesity and inflammation: the linking mechanism and the complications. Arch Med Sci 2017:13:851-63

34 Marques A, Santos E, Nikiphorou E, et al. Effectiveness of selfmanagement interventions in inflammatory arthritis: a systematic review informing the 2021 EULAR recommendations for the implementation of self-management strategies in patients with inflammatory arthritis. RMD Open 2021;7:e001647.

35 Dziura JD, Post LA, Zhao Q, et al. Strategies for dealing with missing data in clinical trials: from design to analysis. Yale $\mathrm{J}$ Biol Med 2013;86:343-58.

36 Matsui T, Kuga Y, Kaneko A, et al. Disease activity score 28 (DAS28) using $C$-reactive protein underestimates disease activity and overestimates EULAR response criteria compared with DAS28 using erythrocyte sedimentation rate in a large observational cohort of rheumatoid arthritis patients in Japan. Ann Rheum Dis 2007;66:1221-6. 